\title{
Machbarkeitsstudie zur Schließung der rehabilitativen Versorgungslücke bei Patient*innen nach erfolgter Majoramputation im Rahmen eines Innovationsfondsprojekts. Umsetzung, Erfahrungen und erste Ergebnisse
}

\section{Feasibility Study on Closing the Rehabilitation Gap in Patients after Major Amputation as Part of an Innovation Fund Project. Implementation, Experiences and First Results}

\section{(ㄷ) (1) (우 $\ominus$}

\section{Autoren}

Christoph Egen $^{1 *}$, Joerg Schiller ${ }^{1}{ }^{*}$, Christoph Gutenbrunner $^{1}$, Isabelle Eckhardt ${ }^{1}$, Katrin Höpner ${ }^{1}$, Christian Sturm ${ }^{1}$, Matthias Fink ${ }^{1}$, Alexander Ranker ${ }^{1}$

\section{Institute}

1 Klinik für Rehabilitationsmedizin, Medizinische Hochschule Hannover, Hannover, Deutschland

\section{Schlüsselwörter}

Amputation, Amputationen der unteren Extremität, ambulante Rehabilitation, interdisziplinäre Rehabilitation, Caremanagement

\section{Key words}

amputation, lower extremity amputation, outpatient rehabilitation, interdisciplinary rehabilitation, caremanagement

eingereicht 26.07.2021

akzeptiert 14.10.2021

online publiziert $\quad 02.12 .2021$

\section{Bibliografie}

Phys Med Rehab Kuror 2022; 32: 218-228

DOI 10.1055/a-1671-3595

(c) 2021. The Author(s).

This is an open access article published by Thieme under the terms of the Creative Commons Attribution-NonDerivative-NonCommercial-License, permitting copying and reproduction so long as the original work is given appropriate credit. Contents may not be used for commercial purposes, or adapted, remixed, transformed or built upon. (https://creativecommons. org/licenses/by-nc-nd/4.0/)

Georg Thieme Verlag, Rüdigerstraße 14,

70469 Stuttgart, Germany
Korrespondenzadresse

Dr. phil. Christoph Egen

Klinik für Rehabilitationsmedizin

Medizinische Hochschule Hannover

Carl-Neuberg-Straße 1

30625 Hannover

Deutschland

Tel.: +49/511/5324 117, Fax: +49/511/5324 130

egen.christoph@mh-hannover.de

\section{ZUSAMMENFASSUNG}

Eine Majoramputation ist für die Betroffenen ein äußerst belastender Eingriff und mit großen Herausforderungen für die gesellschaftliche und berufliche Wiedereingliederung verbunden. Bei der Versorgung von Amputationspatient*innen besteht ein hoher Bedarf an ärztlicher und therapeutischer Behandlung sowie sozialmedizinischer Steuerung. Die sektoralen Versorgungsstrukturen sind regional sehr unterschiedlich aufgestellt und decken häufig den Bedarf nicht adäquat ab. Dem Entlassmanagement kommt hierbei eine Schlüsselposition zu. Ein Innovationsfondsprojekt des Gemeinsamen Bundesausschusses (G-BA) implementiert und evaluiert gegenwärtig modellhaft eine optimierte Versorgung für Menschen nach Majoramputation durch Einführung eines sektorenübergreifenden Caremanagement und der Einführung einer ambulanten Interimsphase in einer Hochschulambulanz für Physikalische und Rehabilitative Medizin (PRM) und Therapie. Der Beitrag berichtet über die Umsetzung, die gemachten Erfahrungen und über erste Ergebnisse.

\section{ABSTRACT}

Major amputation of the lower limb is a stressful and mementous procedure with high challenges for personal, social and occupational reintegration. The complex management of the rehabilitation process of patients after lower limb amputation (LLA)

* geteilte Erstautorenschaft 
needs extensive knowledge in terms of medical issues, therapeutical strategies and social law. Even more, since sectoral structures clearly differ depending on the region and its population density. An innovative project, funded by the German Federal Joint Committee within a program to support innovations in medical supply (Innovationsfonds-Projekt) is evaluating and implementing a new model in the rehabilitation of patients with LLA. The key is to implement a medical care-manager who is helping the patients to get along with the new situation after
LLA by offering different options and managing medical issues. Furthermore, the main goal of the project is to implement an outpatient interim rehabilitation in a university hospital for physical and rehabilitation medicine (PRM) directly after amputation and discharge from the surgical ward. The aim of this paper is to present the experiences, the obstacles as well as the challenges and possibilities of this new approach to postoperative rehabilitation after LLA. Furthermore, first results will be demonstrated.

\section{Hintergrund, Epidemiologie und Problembeschreibung}

Nach einer Amputation der unteren Extremität proximal des Fußes (Majoramputationen ${ }^{1}$; [1]) haben Patient* innen einen sehr hohen individuellen Versorgungs- und Rehabilitationsbedarf, der in Deutschland durch die zu durchlaufenden verschiedenen Sektoren des Gesundheitswesens kaum adäquat gedeckt wird [3]. Nur sehr wenige Akut- und Rehabilitationskliniken sind auf diese Patientengruppe spezialisiert. Gründe für die fehlende Spezialisierung sind die relative Seltenheit von Majoramputationen bezogen auf Fläche und Einwohnerdichte sowie der hohe Aufwand für die benötigte individuelle Versorgung bei vergleichsweise geringen Standard-Vergütungssätzen in der Rehabilitation.

Nach der DRG-Statistik (DRG, Diagnosis Related Groups; diagnosebezogene Fallgruppen) des Statistischen Bundesamts sind im Jahr 2019 in Deutschland insgesamt 16504 Amputationen an der unteren Extremität durchgeführt worden. Die höchste Anzahl an Meldungen mit 2620 Amputationen betraf die Altersgruppe von 80 bis 85 Jahren. Männer wurden deutlich häufiger im Bereich der unteren Extremität amputiert als Frauen (Männer 11204; Frauen 5300). Die transfemorale Amputation stellt weiterhin die häufigste Amputationshöhe dar [4]. Spoden (2019) bestimmte in einer retrospektiven Analyse der OPS (Operationen- und Prozedurenschlüssel) von 2011 bis 2015 eine Inzidenz von 67 Fällen pro 100000 Einwohner in Deutschland - allerdings mit sehr hohen regionalen Unterschieden [5]. Eine Aussage über die Prävalenz kann aufgrund eines in Deutschland noch fehlenden Amputationsregisters nicht getroffen werden.

Ebenso gibt es keine validen Zahlen dazu, wie viele beinamputierte Menschen mit einer Prothese versorgt wurden und welche Gründe für oder gegen diese Versorgung sprachen (fehlende Verordnung, Ablehnung der prothetischen Versorgung von der Krankenkasse usw.). Aus rehabilitativer, versorgungstechnischer und ökonomischer Sicht wäre es darüber hinaus wichtig zu erfahren, ob und wie intensiv vorhandene Beinprothesen genutzt werden. Weiterhin gilt es zu ermitteln, welche Gründe für die Intensität der Nutzung angegeben werden, um Rückschlüsse auf die Versorgungsproblematik zu erhalten. (Wurde das Gehen mit einer Pro-

Gemäß der OPS [2] kann man Majoramputationen wegen des höheren Materialverbrauchs bereits bei der transmetatarsalen Vorfußamputation ansetzen, d. h. von der Fußamputation nach Pirogoff (OPS 5-865.3) bis zur Hemipelvektomie (OPS 5-864.0). these in der Anschlussrehabilitation nicht erlernt? Mangelte es an einer wohnortnahen physiotherapeutischen Gangschule? Sind prothesentechnische Probleme nicht lösbar?) ${ }^{2}$.

- Tab. 1 zeigt eine Übersicht der Amputationen der unteren Extremität, des Fußes sowie der Revisionsoperationen im Zeitvergleich 2019 zu 2009 [4, 6].

Wenngleich die Anzahl der hohen Amputationen der unteren Extremitäten in Deutschland erfreulicherweise rückläufig ist (-26\%), so ist demgegenüber die Gesamtanzahl an Amputationen der unteren Extremitäten im selben Zeitraum leicht gestiegen (+8\%). Dies ist vor allem durch die Zunahme von Fuß- und Zehenamputationen bedingt (+19\%). Auffallend und kritisch zu hinterfragen ist der hohe Anstieg an Revisionsoperationen (+64\%) im betrachteten Zehnjahreszeitraum.

- Tab. 2 zeigt die Verteilung der Amputationshöhen bei Majoramputationen der unteren Extremität im Zeitvergleich 2019 zu 2009 und einen Vergleich nach Geschlechtern.

Hier fällt ebenfalls eine Reduzierung der Amputationszahlen von $25 \%$ und eine Verschiebung um $8 \%$ in der Geschlechterverteilung zu männlichen Patienten auf.

Majoramputierte Patient* innen zeichnen sich durch eine hohe Heterogenität aus. Dies betrifft sowohl die Amputationsursache und damit zusammenhängend die Altersstruktur als auch die Ausprägung der Multimorbidität. Als Ursachen für die Amputation können Trauma, Sepsis, Tumor oder angiologische Erkrankungen genannt werden. In Deutschland ist die Hauptursache für Amputationen nach wie vor die periphere arterielle Verschlusskrankheit (pAVK) und der Diabetes mellitus - oder die Kombination beider. Die Amputationsrehabilitation in Deutschland betrifft daher vornehmlich Patient*innen in einem fortgeschrittenen Alter (> 65 Jahre) $[7,4]$.

Entsprechend der verschiedenen Ursachen sind daher unterschiedliche chirurgische Fachrichtungen für die Amputation verantwortlich (z. B. Gefäßchirurgie, Unfallchirurgie, Tumorchirurgie). Folglich werden auch in den weiteren Behandlungen unterschiedliche Rehabilitationsschwerpunkte gesetzt, obwohl in allen Fällen eine Majoramputation durchgeführt wurde. So kann es sein, dass ein 75-jähriger Patient nach unfallbedingter traumatischer Amputation in einer orthopädischen Rehabilitationsklinik behandelt wird, während ein gleichaltriger Patient bei akutem Gefäßverschluss mit einer Perfusionsstörung (z. B. bei pAVK) in einer Rehabilitations-

2 Es offenbart sich hier - ähnlich wie bei querschnittsgelähmten Menschen - ein eklatanter Mangel an validen Zahlen in Deutschland. 
- Tab. 1 Amputationsstatistik 2009-2019 [4, 6]

\begin{tabular}{|l|l|l|l|l|}
\hline Bezeichnung & OPS-Ziffern & $\mathbf{2 0 0 9}$ & $\mathbf{2 0 1 9}$ & Veränderung (in \%) \\
\hline Amputation und Exartikulation untere Extremität & $5-864$ & 22195 & 16504 & -26 \\
\hline Amputation und Exartikulation Fuß (inkl. Zehenamputation) & $5-865$ & 41363 & 49048 & +19 \\
\hline SUMME & & $\mathbf{6 3 5 5 8}$ & $\mathbf{6 5 5 5 2}$ & $+\mathbf{3}$ \\
\hline Revision eines Amputationsgebietes & $5-866$ & 5738 & 9416 & +64 \\
\hline GESAMTSUMME & & $\mathbf{6 9 2 9 6}$ & $\mathbf{7 4 9 6 8}$ & $+\mathbf{8}$ \\
\hline
\end{tabular}

- Tab. 2 Detaillierte Majoramputationsstatistik 2009-2019 [4, 6]

\begin{tabular}{|c|c|c|c|c|c|c|c|}
\hline & & & & & & \multicolumn{2}{|c|}{ Geschlechtsspezifische Verteilung } \\
\hline \multirow{9}{*}{$\begin{array}{l}\text { Amputalion } \\
\text { Ans }\end{array}$} & & Nr. & Bezeichnung & OPS-Ziffern & Insgesamt 2019 (2009) & Männlich 2019 (2009) & Weiblich 2019 (2009) \\
\hline & & 1 & Hemipelvektomie & $5-864.0 f$ & $71(43)$ & $46(29)$ & $25(14)$ \\
\hline & & 2 & Hüftexartikulation & $5-864.2$ & $154(242)$ & $89(151)$ & $65(91)$ \\
\hline & & 3 & $\begin{array}{l}\text { Transfermorale } \\
\text { Amputation }\end{array}$ & 5-864.3ff. & $9113(12671)$ & $5720(7071)$ & $3393(5600)$ \\
\hline & & 4 & Knieexartikulation & $5-864.7$ & $630(1140)$ & 417 (679) & $213(461)$ \\
\hline & \multirow{4}{*}{$6+\cdots$} & 5 & Transtibiale Amputation & 5-864.8ff. & 6536 (8099) & $4932(5536)$ & $1604(2563)$ \\
\hline & & 6 & $\begin{array}{l}\text { Obere Sprunggelenks- } \\
\text { Exartikulation }\end{array}$ & 5-865.0ff. & $300(378)$ & $225(278)$ & $75(100)$ \\
\hline & & 7 & $\begin{array}{l}\text { Fußamputation nach } \\
\text { Pirogoff }\end{array}$ & $5-865.3$ & $87(75)$ & $65(48)$ & $22(27)$ \\
\hline & & & SUMME & & 16891 (22648) & 11494 (13792) & 5397 (8856) \\
\hline
\end{tabular}

klinik mit internistischem oder geriatrischem Schwerpunkt therapiert wird, gleichwohl die muskuloskelettalen Probleme aufgrund der Amputation überwiegen.

Weiterhin wurde von den Autoren beobachtet, dass Akutkrankenhäuser aufgrund des Verlegungs- und Entlassungsdrucks beinamputierte Patient* innen entweder nach Hause bzw. in die Kurzzeitpflege entlassen und dadurch die notwendige Einleitung der Rehabilitation in die Verantwortung der Hausärztin bzw. des Hausarztes legen (Heilverfahren) oder nahtlos eine stationäre Anschlussrehabilitation (AR/AHB) einleiten. Diese Unterscheidung in der Entlassungs- bzw. Verlegungsart war in den beobachtbaren Fällen nicht indikations-, sondern klinikspezifisch (historisch gewachsene Routine). Ein späterer Zugang zur Rehabilitation (Heilverfahren) hat den Vorteil, dass die Rehabilitand*innen mit einer fertigen Interimsprothese und einem belastbaren Stumpf in die Rehabilitation kommen können. Allerdings birgt dies die Gefahr, dass in der Zwischenzeit häufig keine rehabilitativen Maßnahmen ergriffen werden und es dadurch in besonderen Fällen zu Kontrakturen sowie immobilisationsbedingte Dekonditionierung mit all ihren Folgen kommen kann. Diese Gefahr wird noch durch formal-bürokratische Hürden verstärkt, da der Antrags- und Bewilligungsprozess bei Heilverfahren komplizierter ist als bei einer Anschlussrehabilitation und zudem ggf. durch MDK-Prüfungen verzögert oder abgelehnt wird. Der zweite Zugang (AR/AHB) ist verwaltungstechnisch weniger kompliziert und wird in der Regel von den Kosten- trägern standardmäßig genehmigt, sofern die Rehabilitationsfähigkeit medizinisch bestätigt wurde. Allerdings ist zu beachten, dass zu diesem Zeitpunkt weder die Interimsprothese für die Rehabilitation vorhanden noch der Amputationsstumpf entsprechend belastbar und volumenkonstant ist. Das primäre rehabilitative Ziel, den sicheren Umgang mit einer Prothese einschließlich des Gehens (Mobilität) zu erlernen, kann während des Aufenthaltes in der Rehabilitationsklinik in diesem Fall nur sehr selten erreicht werden. Eine weitere stationäre oder ganztagsambulante Rehabilitation wird üblicherweise erst wieder nach vier Jahren genehmigt. Hier müsste entsprechend eine wohnortnahe anschließende ambulante Versorgung über Therapeut* innen einer physio- und/oder ergotherapeutischen Praxis diese zentralen rehabilitativen Elemente mit den Patient*innen einüben. Es fehlt jedoch speziell in diesem Bereich an bundesweit flächendeckender Kompetenz - dies gilt vor allem hinsichtlich der immer komplexeren elektronischen Prothesensysteme. Zudem würde die ambulante physiotherapeutische Behandlung eine reibungslose und umfassende langfristige hausärztliche Verordnung von Heilmitteln erfordern, was sich aufgrund der hohen Regress-Sorgen und den bürokratischen Hürden als zunehmend schwierig erweist.

Des Weiteren kommen bei dieser zumeist multimorbiden Patientengruppe sehr individuelle Rekonvaleszenzprozesse, z. B. durch Wundheilungsstörungen, starke Stumpfvolumenschwankungen, Hautprobleme an der Naht usw. hinzu, was die Reha-Steuerung 


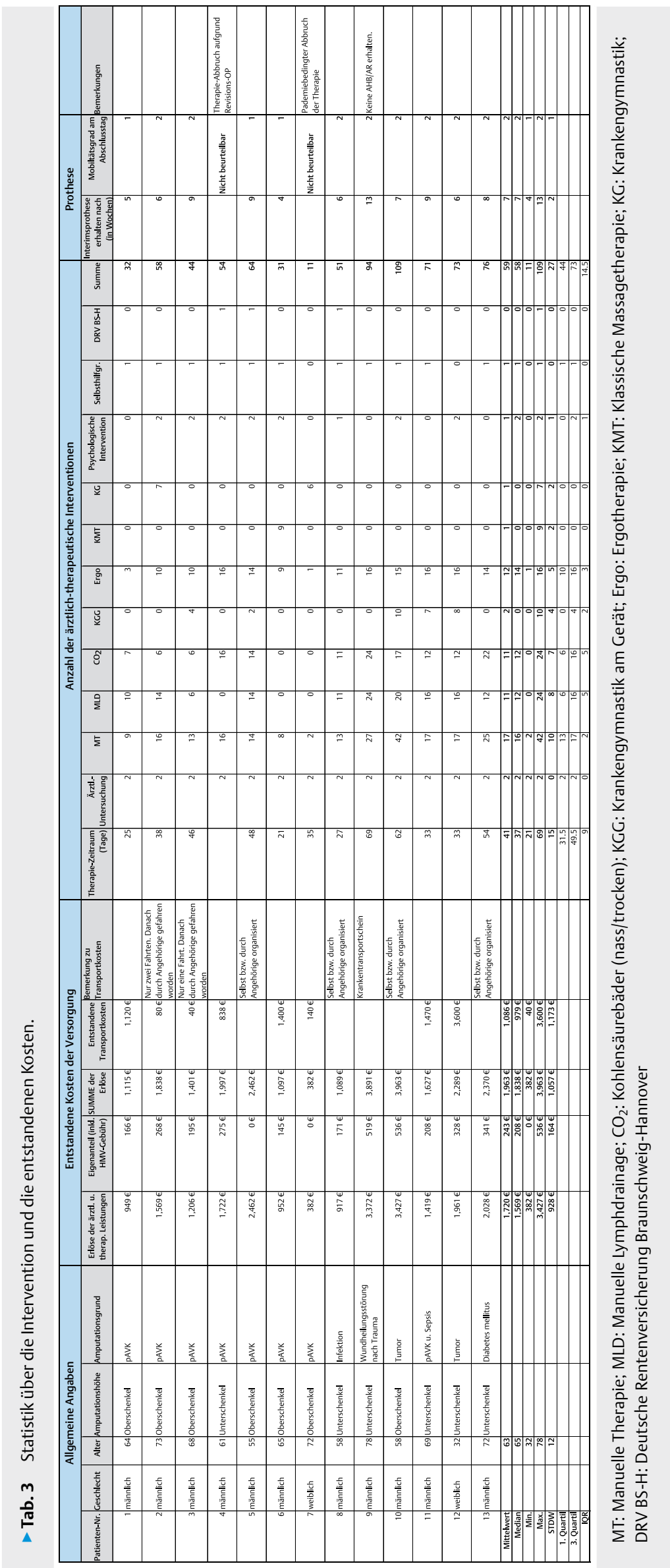


nach den relativ starren Vorgaben der Kostenträger mit einem Antritt der Anschlussrehabilitation spätestens 14 Tage nach Entlassung aus dem Akutkrankenhaus erschwert.

An der fachgerechten Versorgung dieser Patientengruppe sind zahlreiche hoch spezialisierte Gesundheitsberufe beteiligt und viele Sektoren des Gesundheitswesens mit unterschiedlichen Kostenträgern involviert. Die vorhandenen Standardabläufe des Gesundheitssystems liefern hier keine adäquate Antwort auf den medizinischen, sehr individuellen Bedarf der betroffenen Patient*innen - was bereits durch andere Studien sowie Empfehlungen von Fachgesellschaften belegt ist $[3,8,9,10]$.

Zusammenfassend lässt sich festhalten, dass diese Patientengruppe durch folgende Problemstellungen in eine signifikante rehabilitative Versorgungs/ücke [11] fällt:

- unzureichendes Entlassmanagement (z. B. Entlassung in die Kurzzeitpflege, keine Anmeldung zur AR/AHB)

- Verzögerung des Rehabilitationsbeginns (zeitliche Lücke zwischen Akutkrankenhaus und stationärer und/oder ganztagsambulanter Rehabilitation)

- falsches Assignment (Rehabilitationskliniken mit organspezifischer Kompetenz bei geringer bis fehlender Erfahrung im Umgang mit beinamputierten Patient*innen)

- Fehlen einer Kompetenz- und Teamstruktur in der ambulanten, wohnortnahen rehabilitativen Versorgung

- administrative Hürden bei der Genehmigung von Reha- und Heilverfahren

- Unzureichende Berücksichtigung der Rekonvaleszenzphase in der Standardversorgung

- die etablierte, weitgehend standardisierte Rehabilitation gibt keine adäquate Antwort auf die individuellen Versorgungsbedarfe beinamputierter Patient*innen

- hoher Informations-/Aufklärungsbedarf der Patient*innen, der vom Akutbereich nicht gedeckt wird bzw. häufig auch nicht gedeckt werden kann

- kein zentraler Ansprechpartner vorhanden - mit Ausnahme der orthopädietechnischen Fachkräfte - der die Patient*innen über die verschiedenen Sektoren hinweg betreut

Im Rahmen eines vom Innovationsfonds des G-BA's geförderten Projekts „Aufbau und Implementierung eines multimodalen, sektoren- und trägerübergreifenden Versorgungskonzeptes bei Majoramputation (MSTVK)“ (Laufzeit: 01.01.2018 bis 31.12.2021) wurde versucht, diese rehabilitative Versorgungslücke zu schließen [11]. Maßnahmen waren unter anderem die Begleitung und Betreuung der Patient*innen durch ein Caremanagement und die Einführung einer ambulanten Interimsphase zwischen Akutkrankenhaus und stationärer oder ganztagsambulanter Rehabilitation in einer universitären Ambulanz für Physikalische und Rehabilitative Medizin und Therapie. Ziel war, die Patient*innen nach einem interdisziplinärem Assessment ICF-orientiert zu behandeln, um die Rehabilitationsfähigkeit herzustellen und sie zum optimalen Zeitpunkt in eine kooperierende regionale, stationäre oder ganztagsambulante Rehabilitationseinrichtung im Rahmen der Anschlussrehabilitation zu überführen. Der optimale Zeitpunkt für die Anschlussrehabilitation ist aus Sicht der Autoren gekommen, wenn die Rehabilitand* innen mit ihrer vorhandenen Interimsprothese bereits einige Tage mobil sind, eventuelle durch Volumenschwankungen bedingte Anpassungen durchgeführt wurden und der Stumpf belastbar ist. Nur so kann das Kernziel der Rehabilitation, „sicheres Gehen mit der Prothese“, in der anschließenden stationären oder ganztagsambulanten Rehabilitation erreicht werden. Weitere zu vertiefende Reha-Ziele über die Interimsphase hinaus waren die Kontrakturprophylaxe, allgemeine und spezifische Kräftigung der verbliebenen Extremitäten, Erhalt und Ausbau von Koordination und Gleichgewicht sowie Herz-Kreislaufkonditionierung.

Die systematisch aufgearbeiteten Erfahrungen aus diesem Innovationsfondsprojekt werden im Folgenden deskriptiv vorgestellt und daraus Empfehlungen für die Praxis abgeleitet.

\section{Fragestellungen}

- Ist es möglich, Patient* innen mit einem solch komplexen Beschwerdebild direkt nach dem Akutkrankenhausaufenthalt und vor der stationären oder ganztagsambulanten Rehabilitation bedarfsorientiert ambulant rehabilitativ zu behandeln?

- Kann die rehabilitative Steuerung mit Hilfe eines Caremanagements so optimiert werden, dass die Patient*innen zum optimalen Zeitpunkt in die Anschlussrehabilitation kommen?

\section{Inhalte und Vorgehensweise}

\section{Rekrutierungsstrategie}

Die Rekrutierung der Teilnehmer*innen beschränkte sich auf 14 Kliniken für Gefäß- sowie Unfallchirurgie und Orthopädie, die sich in einem Radius von 50 Kilometern um die Hochschulambulanz befanden und einen entsprechenden Kooperationsvertrag unterschrieben haben, sowie zwei regionale orthopädietechnische Unternehmen, die in diesen Krankenhäusern regelmäßig Patient* innen betreuten.

In der Regel meldete die Stationsärztin bzw. der Stationsarzt oder die Mitarbeiter*innen des zuständigen orthopädietechnischen Unternehmens potenzielle Studienteilnehmer*innen, die eine Kontaktaufnahme durch das Caremanagement befürworteten. In seltenen Fällen meldete sich auch der jeweilige Sozialdienst des Akutkrankenhauses. Unmittelbar nach der Amputation und Rückverlegung auf die Normalstation wurde ein persönlicher Termin vereinbart und ein erstes Informationsgespräch geführt. Wenn alle Einschlusskriterien erfüllt waren ( $\triangleright$ Tab. 4), erhielten die Teilnehmer*innen der Studie ein Informationsschreiben und unterschrieben die Teilnehmererklärung. Alle Patient*innen, deren Wohnort weniger als 50 Kilometer von

- Tab. 4 Ein- und Ausschlusskriterien.

\begin{tabular}{|l|l|}
\hline Einschlusskriterien & Ausschlusskriterien \\
\hline - Majoramputation an einer der & - Amputation im Ausland \\
& unteren Gliedmaßen \\
- vor der Amputation gehfähig & - mehrfachamputiert \\
- über 18 Jahre alt & - Patient*innen, die unter \\
- ausreichende Deutschkenntnisse & $\begin{array}{l}\text { gesetzlicher Betreuung } \\
\text { stehen }\end{array}$ \\
\hline - zum Ausfüllen des Fragebogens & \\
\hline
\end{tabular}


der Hochschulambulanz entfernt war und die in der Lage waren, mit Angehörigen, einem Taxi oder in Ausnahmefällen mit einem Krankentransport den Transfer zwischen Wohnort und Hochschulambulanz durchzuführen, wurden in die Interventionsgruppe aufgenommen. Alle anderen kamen in die Kontrollgruppe und durchliefen die Regelversorgung.

\section{Caremanagement}

Die Personen des Caremanagements (bestehend aus einer Physiotherapeutin und einem auf Amputationsrehabilitation spezialisierten PRM-Arzt) dienten vom Akutkrankenhaus bis zur Langzeitversorgung als feste Ansprechpartner*innen für die Patient*innen. Dies beinhaltet im Einzelnen:

- die Kontaktaufnahme im Akutkrankenhaus

- die Terminkoordination für die ambulante Interimsversorgung

- die Klärung des besten Transportweges zur ambulanten Interimsversorgung

- Kontaktaufnahme zu dem von den Patient*innen gewählten orthopädietechnischen Unternehmen (falls nicht bereits vom Akutkrankenhaus aus initiiert)

- Einleitung der AR/AHB selbstständig oder in Zusammenarbeit mit dem entsprechenden Sozialdienst des Akutkrankenhauses

- Kontaktaufnahme zur Krankenkasse, um a.) den gewählten Zeitpunkt (4-6 Wochen nach der Amputation) zur Anschlussrehabilitation genehmigen zu lassen bzw. abzusichern und über die Studie zu informieren und b.) die Krankenkasse davon zu überzeugen, den Aufenthalt in einer der beiden kooperierenden Rehabilitationskliniken zu genehmigen

- informative Unterstützung der Betroffenen (z. B. Beantragung eines Behindertenausweises, Beantragung eines europaweiten Schlüssels für den Zugang zu Behinderten-WCs auf Raststätten, Beantragung einer geänderten KFZ-Fahrerlaubnis, Information über Selbsttrainingsprogramme und entsprechende Apps, Aushändigung des „AmpuRucksacks“ (Erste-Hilfe-Rucksack) des Bundesverbands für Menschen mit Arm- oder Beinamputation, Empfehlungen zum Wohnungsumbau und zur Beantragung entsprechender Hilfsmittel)

- wenn gewünscht: Vermittlung des Kontakts zur Selbsthilfegruppe für Amputierte in der Region Hannover

- bei Berufstätigkeit und Bedarf: Vermittlung der Fachberatung Rehabilitation der Deutschen Rentenversicherung (Bund oder Braunschweig-Hannover) zur ggf. Einleitung unterstützender Maßnahmen für den Wiedereinstieg in den Beruf oder zur Übernahme der Kosten einer Umschulung

- ggf. Informationsweitergabe über wohnortnahe Sport-/ Trainingseinrichtungen

- Besuch während bzw. nach der Anschlussrehabilitation zur Abklärung eines ggf. weiteren Versorgungsbedarfs

- ggf. Koordination weiterer Therapietermine in der Universitätsklinik, beispielsweise Gehschulung

- Erstellung von Informationsmaterialen (Selbstübungsprogramme sowie ein regionaler Ratgeber)

\section{Ambulante ärztlich-therapeutische Versorgung während der Interimsphase}

Die Interimsphase fand in einer Hochschulambulanz für Physikalische und Rehabilitative Medizin (PRM) und Therapie statt. Die beteiligten Professionen waren:

- Fachärzt*innen für Physikalische und Rehabilitative Medizin (PRM)

- Physiotherapeut*innen

- Ergotherapeut*innen

- Hydrotherapeut*innen

- Psycholog*innen

- Externe Orthopädietechniker*innen

- Peers im Krankenhaus (PiK)

- Bei Bedarf: individuelle Berufsberatung der Deutschen Rentenversicherung Braunschweig - Hannover

Bei Studieneinschluss erhielten die Patient*innen direkt im Anschluss an den Akutkrankenhausaufenthalt einen Termin in der Hochschulambulanz zur Durchführung eines ICF-orientierten, multimodalen Assessments unter ärztlicher Leitung. Der ärztliche Teil des Assessments bestand aus einer umfassenden Anamnese (inkl. Erfragung sämtlicher Barrieren sowie Unterstützungsmöglichkeiten im Umfeld) und klinischer, amputationsspezifischer Untersuchung. Anschließend wurde über Hilfsmittel informiert und der Bedarf evaluiert. Objektive Messparameter bestanden aus dem AmpuNoPro [12], einem standardisierten klinischen Test zur Evaluation der prognostizierten Mobilität. Weiterhin wurden der FIM (Functional Independence Measure) [13] sowie zwei weitere Tests zur Evaluation der Handkraft und der Stützkraft im oberen Rumpf und der Oberarme durchgeführt. Im Anschluss wurden individuelle Rehabilitationsziele gemeinsam definiert und entsprechend individuelle zielgerichtete physikalisch-medizinische Therapien mit einer Frequenz von 2-3mal wöchentlich verordnet (Heilmittelverordnung). Die Aufenthaltsdauer der Patient*innen variierte an diesen Tagen zwischen 3 und 5 Stunden.

Folgende Versorgungselemente wurden (optional) einbezogen:

- ärztliche Diagnostik, Screening, Therapiesteuerung und -verlaufskontrolle

- Schmerztherapie, Einstellung der Medikation, Prävention von Phantom- und Stumpfschmerz

- manuelle Therapie (MT) zum Erhalt der Gelenkfunktionen und zur Reduktion von Kompensationsstörungen

- komplexe Entstauungstherapie mit manueller Lymphdrainage (MLD) und individueller Kompressionsbehandlung

- $\mathrm{CO}_{2}$-Therapie zur Förderung der Wundheilung der Stumpfnaht

- aerobes Ausdauertraining und Krankengymnastik am Gerät (KGG) zur Kräftigung der Muskulatur, Optimierung der Koordination und Erhalt der Flexibilität

- Ergotherapie, um den Umgang mit der Prothese zu erlernen, Schmerzen vorzubeugen oder diese zu behandeln, z. B. mittels Spiegeltherapie, motorisch funktioneller Behandlung sowie ADL-Training und Hilfsmittelberatung

- klassische Massagetherapie (KMT) zur Verbesserung der Zirkulation und Schmerzreduktion, Entspannung sowie als Segment- und Reflextherapie 
- Gespräch mit einem Vertreter der Selbsthilfegruppe

- Psychologische Intervention (2 Einheiten)

- Gespräch mit einem Berater der DRV BS-H

Während der ambulanten multimodalen Therapie wurde ärztlicherseits die nachfolgende ganztagsambulante oder stationäre Anschlussrehabilitation organisiert, falls dies nicht nach Absprache mit dem Sozialdienst des jeweiligen Akutkrankenhauses bereits geschehen war, und stets der Kontakt zu weiteren gesundheitsdienstlichen Sektoren gehalten (Orthopädietechnik, Chirurgie, Hausarzt, usw.). Vor Antritt dieser Phase wurden in einer Abschlussuntersuchung die Rehabilitationsfähigkeit und die Rehabilitationsziele erneut evaluiert. In manchen Fällen musste der Antrittstermin zur AR/AHB verschoben werden, was eine enge Kommunikation mit den Leistungsträgern und der Leistungserbringern erforderte.

Bei Bedarf konnten weitere Arzt- und Therapietermine nach der $A R / A H B$ vereinbart werden, z. B. für eine weiterführende Gangschule.

\section{Erste Ergebnisse und Erkenntnisse aus der Praxis}

Es wurden 71 potenzielle Studienteilnehmer*innen innerhalb des vorgesehenen Rekrutierungszeitraums vom 01.04.201831.12.2020 durch die 14 kooperierenden Akutkliniken gemeldet. Davon konnten 39 Patient*innen in die Kontrollgruppe (KG) und 13 in die Interventionsgruppe (IG) aufgenommen werden. Der Hauptausschlussgrund zur Teilnahme an der IG war eine Distanz vom Wohnort zur Hochschulambulanz von mehr als 50 Kilometern wegen der zu hohen Transportkosten. Der häufigste Grund, warum Patienten weder in die KG noch IG eingeschlossen wurden, war unzureichende psychisch-physische Fähigkeit, z. B. durch Demenz oder neurologische Begleiterkrankungen.

Alle Teilnehmer*innen erhielten in regelmäßigen Abständen (im Akutkrankenhaus, nach der Interimsphase, nach der AR/AHB, 6 und 12 Monate nach AR/AHB) validierte Fragebögen zur Lebensqualität (SF-12; [14]), prothetischen Versorgung (PEQ; [15]), Teilhabeeinschränkung (IMET; [16]), subjektiven Prognose der Erwerbsfähigkeit (SPE; [17] ), Arbeitsfähigkeit (WAI; [18]), Patientenzufriedenheit (ZUF; [19]) und weitere Fragebögen durch die unabhängige Begleitevaluation der Universität zu Lübeck (Prof. Dr. phil. Matthias Bethge). Ebenfalls wurden von dort persönliche Interviews mit den Patient*innen und mit Vertreter*innen der verschiedenen Berufsgruppen geführt. Da die offizielle (Nach-)Evaluation noch bis zum 31.12.2021 läuft, liegen die Evaluationsergebnisse erst im Sommer 2022 vor.

\section{Soziodemographische und anthropometrische Daten}

Von den 13 Studienteilnehmer* innen waren 11 männlich und 2 weiblich. Der Altersdurchschnitt lag bei 63 Jahren (Range: 32-78 Jahre; Stdw.: 12 Jahre). Gründe der Amputation war bei $61 \%$ der Patient*innen eine arterielle Verschlusserkrankung (pAVK), bei $15 \%$ ein Tumor und bei je $8 \%$ Diabetes, Infektion und Wundheilungsstörung. Bei $54 \%$ erfolgte eine Oberschenkel- und bei $46 \%$ eine Unterschenkelamputation. Bei vier Teilnehmer* innen lag eine Wundheilungsstörung vor. Neun Teilnehmer* innen hatten vor der
Amputation keinen Pflegegrad, jeweils zwei Teilnehmer*innen hatten bereits vorher einen Pflegegrad 2 bzw. 3.

Bis auf zwei Personen durchliefen alle Studienteilnehmer*innen die gesamte Interimsphase. Bei einer dieser Personen war die Ambulanz aufgrund der Corona-Hygiene-Maßnahmen geschlossen und bei der anderen kam es während dieser Phase zu mehrfachen Revisionsoperationen. Ein Versuch der Kontaktaufnahme zur zweiten Person zur Weiterführung der Therapie scheiterte an deren Nichterreichbarkeit. Eine dritte Person erhielt keine stationäre bzw. ganztagsambulante Anschlussrehabilitation, da laut MDK-Prüfung keine Rehabilitationsfähigkeit vorlag. Bei dieser Person wurde die Interimsphase verlängert, so dass sie vollständig ambulant den Umgang und das Gehen mit der Prothese erlernte.

\section{Ärztlich-Therapeutische Interventionen}

Die ambulante Interimsphase dauerte durchschnittlich 41 Tage (Range: 21-69 Tage; Stdw.: 15 Tage). Die behandelten Patient*innen erhielten im Durchschnitt 59 ärztlich verordnete Therapieeinheiten bei Bedarf inkl. psychologischer Gespräche, Selbsthilfegruppe und Berufsberatung (Range: 11-109 Einheiten; Stdw.: 27 Einheiten). Die eingesetzten Heilmittel waren MT, KG, KGG, MLD, KMT, $\mathrm{CO}_{2}$ und sensorisch-perzeptive Ergotherapie ( $\triangleright$ Abb. 1 und $\triangleright$ Tab. 3). Zusätzlich zu den Therapieeinheiten gab es eine ärztliche Eingangs- und Abschlussuntersuchung (inkl. Assessment), Teambesprechungen und regelmäßige ärztliche Therapievisiten. Im gesamten Zeitraum erfolgten regelmäßige Teambesprechungen und Therapievisiten. Bei volumenkonstantem Stumpf wurde in enger Zusammenarbeit mit dem jeweiligen orthopädietechnischen Unternehmen innerhalb dieser Zeit die Interimsprothese angepasst und fertiggestellt. Im Durchschnitt lagen ca. 7 Wochen zwischen der Operation und fertiggestellter Interimsprothese (Range: 4-13 Wochen; Stdw.: 2). Der überwiegende Teil der Teilnehmer*innen verfügte am letzten Tag der ambulanten Intervention über einen Mobilitätsgrad von 2 (eingeschränkter Außenbereichsgeher). Von den Oberschenkelamputierten erhielten vier ein elektronisches und zwei ein mechanisches Kniegelenk.

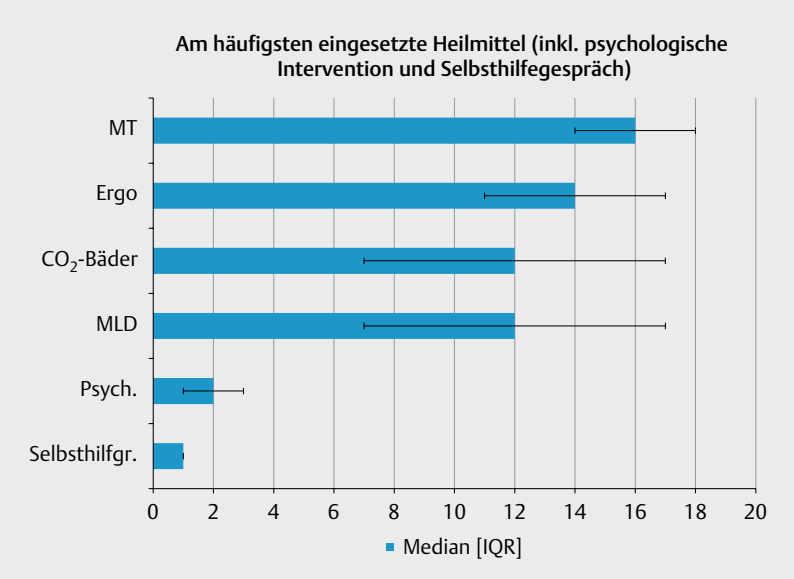

- Abb. 1 Am häufigsten eingesetzte Heilmittel inkl. psychologische Intervention und Gespräch mit der Selbsthilfegruppe. MT: Manuelle Therapie; Ergo: Ergotherapie; MLD: Manuelle Lymphdrainage; Psych.: Psychologische Intervention; IQR: Interquartilsabstand 


\section{Klinische Ergebnisse}

Diese Studie zeigte durch die unmittelbare engmaschige Versorgung in der postoperativen ambulanten Phase klinische Schwerpunkte, die hoch priorisiert werden sollten, erfahrungsgemäß aber meist zu kurz kommen.

In der postoperativen Phase nach Entlassung zeigte sich vorerst die Wundheilungsstörung bei den gängigen Komorbiditäten dieses Patientenklientels als primärer Fokus. Eine abgeschlossene Wundheilung ist Grundvoraussetzung für eine möglichst unterbrechungsfreie Trainingsphase bei den ersten Gehversuchen mit Prothese. Physikalisch-medizinische Verfahren wie trockene Kohlendioxid-Bäder, regelmäßige Debridements, angepasste Kompressionstherapie sowie Reduktion des Lymphstaus proximal der Amputation (z. B. Aktivierung inguinal) zeigten meist rasche Wirkung und förderten die Wundheilung. Um eine schnelle Mobilisierung zur Teilhabe in der Rehabilitation voranzutreiben, sind hier weitere physikalische Maßnahmen indiziert.

Ein weiterer klinischer Fokus, der durch diese Intervention an Sichtbarkeit gewann, ist die frühzeitige Kräftigung der Rumpf- und Schultergürtelmuskulatur und der oberen Extremitäten, um eine ausreichende Stützkraft für das folgende Gangtraining zu erzielen. Die erste Phase des prothetischen Ganges ist maßgeblich an die Sicherheit der Bewegung mit den Gehhilfen gekoppelt, ebenso der Transfer von Bett zu Rollstuhl oder umgekehrt. Die muskuloskelettale Funktionsfähigkeit des Stütz-und Halteapparats sollte sehr früh evaluiert werden, um rehabilitationshindernde Barrieren zu erkennen. Die interdisziplinären Eingangs-Assessments helfen dabei, etwaige Defizite frühzeitig sichtbar zu machen und gegenzusteuern.

Die Schmerztherapie mit fortlaufender Anpassung der Medikation und engmaschiger Evaluation der Schmerzen sollte ebenso beobachtet werden. Häufig ist die Entlassmedikation mit hohen Dosen an schwachen Opioden rasch reduzierbar. Dies fördert die Propriozeption und die posturale Kontrolle für das kommende Prothesengangtraining und reduziert die Gefahr einer Abhängigkeitsund Toleranzentwicklung. Auf der anderen Seite sind Phantomschmerzen oder lokale neuropathische Schmerzen frühzeitig zu beachten und entsprechend zu behandeln (z. B. Antikonvulsiva, trizyklische Co-analgetika oder Calcitonin) und die physikalischen Therapieverfahren frühzeitig daran anzupassen.

\section{Ökonomische Aspekte}

Die innovative ambulante Intervention erzeugte im Durchschnitt $1720 €$ Kosten pro Patient ${ }^{*}$ in, die durch die Krankenkasse übernommen wurden. Der von Patient*innen in der Regel zu leistende Eigenanteil in Höhe von 10 \% zusammen mit den Gebühren pro Heilmittelverordnung ergab eine durchschnittliche Summe von $243 €$ Eigenanteil pro Patient* in. Hohe Kosten verursachte in einigen Fällen der Transport vom Wohnort zur Universitätsklinik. 5 Personen organisierten den Transport in die Ambulanz selbstständig (Familienangehörige, öffentliche Verkehrsmittel). 8 Personen nahmen den Transport durch ein Taxi in Anspruch. Hier lagen die mittleren und vom Innovationsfond getragenen Kosten bei $1086 €$.

Hinzuzurechnen ist das Caremanagement, das in Form von Stellenanteilen vom Innovationsfonds übernommen wurde.

- Tab. 3 zeigt die Anzahl der abgegebenen ärztl.-therapeutischen Interventionen sowie deren Kosten. Weiterhin sind die Transportkosten sowie weitere soziodemographische Angaben ablesbar.

\section{Zusammenfassung und Limitationen}

Die Erfahrungen zeigen, dass eine solche multimodale ambulante Therapie in einer Universitätsklinik implementierbar ist und die rehabilitative Versorgungslücke zwischen Akutklinik und Anschlussrehabilitation, in die viele beinamputierte Menschen aufgrund der o. g. Problematiken fallen, effektiv geschlossen werden kann. Auch wenn diese Interimsphase zunächst einmal Kosten verursacht, liegt die Vermutung nahe, dass das Gesundheitssystem langfristig durch eine bedarfsorientierte Versorgung Kosten einspart, etwa durch Vermeidung von „Arzt-Hopping“ und Rehabilitationsmaßnahmen zu einem ungünstigen Zeitpunkt oder durch Vermeidung prothetischer Fehlversorgung und Chronifizierungen durch gezielt eingesetzte Kompetenz. Ebenso könnte ggf. eine geringere Komplikations- und Revisionsrate erzielt werden. Eine genauere Untersuchung und Evaluierung hierzu steht allerdings noch aus.

Das Caremanagement entlastete die Teilnehmer*innen durch die Übernahme administrativer Aufgaben und koordinierte den Gesamtprozess. Hierzu war ein hoher kommunikativer Aufwand notwendig. Generell scheint Kommunikation auch ein wesentlicher Erfolgsfaktor zu sein, um Schnittstellenprobleme zu lösen. So zeigen die Erfahrungen, dass auch Kranken- und Rentenversicherungen einen gewissen Spielraum haben, wenn es beispielsweise um einen späteren Antritt der AR/AHB geht - dies aber funktioniert nur durch persönlichen Austausch und nicht über Formulare. In einem einzigen Fall wurde diesbezüglich kein Konsens gefunden und die AR/AHB aufgrund einer aktenbasierten nicht vorhandenen Rehabilitationsfähigkeit abgelehnt. Interessanterweise konnte trotz nicht vorhandener Rehabilitationsfähigkeit - jener Patient komplett ambulant im Rahmen der Ausdehnung der Interimsphase rehabilitiert werden. Alle anderen Patient*innen konnten mittels angepasster Interimsprothese deutlich schmerzreduziert und rehabilitationsfähig die geplante Rehabilitation antreten und dort von den weiterführenden Maßnahmen profitieren.

Dem gegenüber stehen auf der anderen Seite erhebliche Kosten und mehrere Barrieren. Die größte Barriere bildet in diesem Modul der Transport der Teilnehmenden zwischen Wohnung und Hochschulambulanz. Zwar könnten die Akutkrankenhäuser - wenn indiziert - einen Eilantrag auf einen Pflegegrad 3 stellen (was unserer Erfahrung nach i.d.R. nicht erfolgt), allerdings sichert auch ein Pflegegrad 3 paradoxerweise keine Transportkostenübernahme bei Teilnahme an einer klinischen Studie, unabhängig vom Inhalt (manche Kassen übernahmen diese nach Rücksprache dennoch). Hier wurde Abhilfe geschaffen, indem der Innovationsfonds die Transferkosten (Krankentransport und/oder Taxi) nach realer Abrechnung übernahm. Diese Fahrten können je nach Entfernung zum Wohnort sehr hohe Kosten innerhalb dieser poststationären ambulanten Phase verursachen ( $\triangleright$ Tab. 3) und stellen daher für eine Übernahme dieser Versorgungsform eine finanzielle Hürde dar.

Ein weiteres Problem sind die Zuzahlungen von 10 \% des Rezeptwertes bei den Heilmitteln (Therapien) sowie die Rezeptgebühr in Höhe von $10 €$ pro Heilmittelverordnung (HMV). Diese haben beispielsweise innerhalb der Interimsphase Kosten bis zu $536 €$ verursacht. Dies führte in unserem Fall (z. B. Rentner*in) zu erheblichen finanziellen Belastungen, sofern keine Befreiung vorliegt.

Für die Interimsversorgung bestanden Kooperationen mit der Selbsthilfegruppe für Amputierte der Region Hannover, der Deutschen Rentenversicherung Braunschweig-Hannover, zwei ortho- 
> Tab. 5 Empfehlungen

\section{Empfehlungen für eine bedarfsgerechte klinische Versorgung von beinamputierter Menschen in der Interimsphase zwischen Akutkrankenhaus} und AR/AHB oder als Ersatz für eine AR/AHB

Organisation

peutische

Intervention
- Einsatz eines zentralen sektorenübergreifenden bzw. -unabhängigen Caremanagements

- Einsatz von geschulten Peers die beratend den Patient*innen zur Seite stehen

- Evaluation des Settings gemäß ICF inklusive Umweltfaktoren, Aktivitäten und Partizipation für individuelle Rehabilitationszielplanung

- Regelmäßige ärztliche Kontrolle (Rehabilitationssteuerung, Heilmittelverordnungen)

- Stumpfbehandlung

a) Förderung der Wundheilung (z. B. mit physikalisch-medizinischen Verfahren wie $\mathrm{CO}_{2}, \mathrm{MLD}$, Mikrostrom, usw.)

b) Förderung der Stumpfformung ggf. durch engfrequentierte Wickelungen

c) Postoperative Schmerztherapie inkl. frühzeitige Phantomschmerz-evaluation und ggf. Therapie

d) Sensorisch-perzeptive Ergotherapie zur Desensibilisierung des Stumpfendes und Phantomschmerzprophylaxe (z. B. Spiegeltherapie)

- Physiotherapie, insbesondere Kontrakturprophylaxe und Stützkraft-training, Rumpfstabilität, Hilfsmittel-Anleitung (besonders Gehen mit Unterarmgehstützen)

- Motorisch-funktionelle Ergotherapie mit Einübung zum Prothesenhandling und Durchführung von Aktivitäten des tägl. Lebens

- Angebot zur psychotherapeutischen und/oder psychologischen Unterstützung pädietechnischen Unternehmen sowie einem niedergelassenen Psychologen bzw. der Klinik für Psychosomatik und Psychotherapie der Medizinischen Hochschule Hannover (MHH). Die Koordinierung dieser Kooperationen war ebenfalls Teil des Caremanagements.

Auch wenn es keine objektiven Parameter gibt, um die eingangs gestellte Frage nach der Möglichkeit der Schließung der rehabilitativen Lücke bedenkenlos zu beantworten, zeigen die Projekterfahrungen, dass das vorgestellte Modell durchaus in der Lage wäre, diese Lücke zumindest deutlich zu reduzieren, wenn nicht sogar potenziell ganz zu schließen.

Die geringe Anzahl der Probanden lässt selbstverständlich keine endgültige Aussage zu, sondern kann lediglich modellhaft die Instrumente und Vorgehensweisen zur Lösung des Problems demonstrieren. Gleichzeitig muss darauf hingewiesen werden, dass eine solche ambulante Interimsphase nur für eine Patientengruppe in Frage kommt, die über ein entsprechend gut organisiertes Umfeld und eine gute Compliance (Kontextfaktoren) verfügt - und diese Gruppe ist bei Majoramputationen, wie die Erfahrung zeigt, relativ klein. Für die deutlich größere Gruppe von majoramputierten und multimorbiden, meist geriatrischen Patient*innen wäre die Einführung einer Reha-Phase C, die bislang nur in der neurologischen Rehabilitation vorgehalten ist, sinnvoll. Die Öffnung dieser Phase für Trauma-Patient* innen ist bereits seit einiger Zeit in der Diskussion [20, 21]. Hier bietet sich die Möglichkeit weitere Patientengruppen, wie beispielsweise Majoramputierte, ebenfalls zu inkludieren.

\section{Ausblick}

Maßnahmen der mit öffentlichen Mitteln finanzierten Innovationsfondsprojekte, die positiv evaluiert wurden und nach entsprechendem Votum des G-BA's, sollten ganz oder teilweise in die Regelversorgung übernommen werden bzw. „als Grundlage für strukturelle Veränderungen des gesetzlichen Rahmens dienen“ [22]. Auch wenn das Ergebnis der externen Evaluation in diesem Fall noch nicht vorliegt, kann bereits heute davon ausgegangen werden, dass Maßnahmen des vorliegenden Projekts - auch bei noch so guter Evalu- ation - nicht in die Routineversorgung übernommen werden. Um eine Verstetigung beispielsweise in Form eines IV-Vertrags nach § 140a SGB V vor ihren Aufsichtsräten zu legitimieren, benötigen Krankenkassen einen sogenannten Business-Case, der wiederum größere Fallzahlen zur Validierung braucht. Die wenigen Hochschulambulanzen der Physikalischen und Rehabilitativen Medizin und Therapie in Deutschland verfügen jedoch in der Regel nicht über die erforderlichen personellen Kapazitäten für die intensive Rehabilitation eines größeren Patientenkollektivs von beispielsweise 100 oder mehr beinamputierten Patient*innen pro Jahr. Diese Kapazitäten müssten mithin geschaffen, also finanziert werden, die kostendeckende Finanzierung hängt allerdings wiederum an dem IV-Vertrag.

Wie häufig in drittmittelgeförderten Projekten gibt es zwar einen Erkenntnisgewinn, allerdings - mangels Übernahme in die Routineversorgung - ohne dass Patient*innen langfristig davon profitieren. Es hat den Anschein, dass es in Deutschland durch entsprechende Förderung zwar einzelne regionale Leuchtturmprojekte (z. B. Exoprothesennetz Schleswig-Holstein) gibt, aber keine Bestrebungen erkennbar sind, flächendeckend eine Optimierung der Versorgung beinamputierter Menschen vorzunehmen. Leider wird es zu einer Optimierung vermutlich erst kommen, wenn die Fallzahlen durch Krisen und/oder Volkskrankheiten ansteigen.

\section{Erfahrungen und Empfehlungen}

Die hohe Zahl der durchgeführten Therapien und deren Frequenz, sowie die regelmäßigen ärztlichen Visiten während der ambulanten Interimsphase zeigen einen medizinisch-therapeutischen Bedarf auf, der in der Regelversorgung nicht erfüllt wird. Einerseits zeigt die Dauer des Behandlungszeitraums, dass eine zu frühe AHB nicht sinnvoll ist. Bei den evaluierten Patient*innen wäre der früheste Zeitpunkt 5 Wochen nach Amputation gewesen (Fertigstellung der Interimsprothese nach 4 Wochen, nachfolgend 1 Woche Übung, dann Zugang in die AR/AHB) und der späteste 14 Wochen nach Amputation. Die durchschnittliche Dauer lag bei 7 Wochen. Andererseits lässt die Dauer des Behandlungszeitraums vermuten, 
dass eine Phase der Immobilisierung - wie durch die Verlegung nach Hause oder in die Kurzzeitpflege - massive Schäden bei den Amputierten erzeugen und unter Umständen auch zu einer dauerhaften Immobilität/Pflegebedürftigkeit führen kann.

Hochschulambulanzen für Physikalische und Rehabilitative Medizin und Therapie sind geeignet auf die sehr individuellen medizinischen Bedarfe beinamputierter Menschen einzugehen ${ }^{3}$. Sie können sowohl eine rehabilitative Interimsversorgung gewährleisten als auch - bei entsprechender Verlängerung des Behandlungszeitraums - eine stationäre oder ganztagsambulante Rehabilitation vollständig substituieren ( $\triangleright$ Tab. 5), wodurch Kostenträger durchaus öffentliche Mittel einsparen könnten bei gleichzeitiger Verbesserung der Versorgung. Allerdings findet die Interimsphase immer zu Lasten der gesetzlichen Krankenversicherung statt. Zwar ist diese - aufgrund des in der Regel höheren Alters beinamputierter Menschen - generell Hauptkostenträger, andererseits gibt es auch berufstätige Menschen, die in ihrer Freizeit einen Unfall mit Amputationsfolge haben oder die aufgrund einer Erkrankung beinamputiert werden. Hier ist folglich die Deutsche Rentenversicherung in der Pflicht, die wiederum aufgrund der gesetzlichen Vorgaben keine Kosten in einer Hochschulambulanz übernehmen kann.

Die zusätzlichen patientenseitigen Kosten, die Transportanforderungen und die Finanzierung eines Caremanagers stellen hier die größten Umsetzungsbarrieren dar.

Die auch gesetzlich verankerte sektorenübergreifende Zusammenarbeit offenbart sich in diesem wie in vielen anderen Fällen als Utopie, die durch alternierende gesetzliche Vorgaben keine Chance auf Realisierung hat. Die Komplexität der Grundvoraussetzungen der Patient*innen in Kombination mit den unterschiedlichen Trägern und Sektoren kann nur durch die Installierung eines verbindenden, kommunikativen Zwischengliedes aufgelöst werden. Diese Erkenntnis unterstreicht die Bedeutung des Caremanagements. Die Caremanagerin bzw. der Caremanager bildet durch spezielle Kompetenz und das Wissen über die notwendigen Handlungsschritte einen zentralen Ankerpunkt für Patient*innen nach Amputation im Rehabilitationsprozess.

Hieraus lassen sich für die Versorgungsrealität aus Sicht der Autoren folgende Empfehlungen ableiten:

- Einführung eines spezifischen Caremanagements als krankenhausübergreifender Ansprechpartner für eine definierte Region oder als überregionale Kontaktstelle, auch niederschwellig durch sozialmedizinische Schulungen/Zertifizierung von Peers denkbar

- Zentrenbildung nach dem Vorbild der Querschnittzentren, die sowohl den akut- als auch den rehabilitationsmedizinischen Bereich für querschnittgelähmte Menschen abdecken (nach dem BG-Konzept „alles aus einer Hand“) oder nach dem Vorbild des Zentrums für Integrierte Rehabilitation (ZIR) in den Universitäts- und Rehabilitationskliniken Ulm (RKU), in

3 Selbstverständlich könnten auch ambulante Rehabilitationszentren eine solche Interimsphase anbieten, wenn die fachlichen Voraussetzungen gegeben sind. Allerdings müssten sich zuerst die gesetzlichen Rahmenbedingungen ändern, damit eine solche vorgeschaltete oder alternierende Reha-Leistung von den Kostenträgern übernommen wird. Als Standard-Rehabilitation kann die Interimsphase nicht verordnet werden, da in den meisten Fällen keine ausreichende Rehabilitationsfähigkeit im Sinne einer Anschlussheilbehandlung bei diesen Patient*innen vorliegt. der sich ebenfalls Akut- und Rehabilitationsbereich unter einem Dach befinden

- Zentrenbildung in Universitätskliniken in Form von z. B. Tageskliniken um Leistungen, die nicht im Heilmittelkatalog vorgesehen sind, aber benötigt werden, zu refinanzieren und/ oder als RehaKompetenzCentren (RKC) als Ansprechpartner für Probleme, die im niedergelassenen Bereich nicht gelöst werden konnten

- Schulungen für niedergelassene Ärzt*innen (Allgemeinmedizin, Orthopädie und Unfallchirurgie, Orthopädie, Chirurgie mit spezieller Unfallchirurgie, Physikalische und Rehabilitative Medizin) sowie für Physio- und Ergotherapeut*innen, um flächendeckende rehabilitative Kompetenzen für die Langzeitversorgung zu generieren

- Darüber hinaus wäre eine Schaffung von DRG-Anreizen zur interdisziplinären Zusammenarbeit bereits vor der Amputation (Chirurgie, Rehabilitationsmedizin, Orthopädietechnik und Physiotherapie) wünschenswert, um ein für den Patienten bestmöglichstes Ergebnis zu erzielen ${ }^{4}$.

\section{Danksagung}

Die Autoren bedanken Sie bei den Patient*innen für die Teilnahme an der Studie sowie bei Frau Katharina Reineck und Frau Inga Bannier aus der ambulanten Patientenabrechnung der Medizinischen Hochschule Hannover für die Zurverfügungstellungen der ökonomischen Daten.

\section{Interessenkonflikt}

Die Autorinnen/Autoren geben an, dass kein Interessenkonflikt besteht.

\section{Literatur}

[1] Greitemann B, Bork H, Braatz F, Büttner CC, Brückner L, Grünther RA, Kaiser H, Kokegei D, Middeldorf S, Möller M, Panning S, Rohland D, Schröter J, Seckler M, Stein N, Sonnenberg D. (2019). Leitlinie eines speziellen Rehabilitationskonzeptes. Rehabilitation nach Majoramputation (proximal des Fußes), AWMF Leitlinienregister Nr. 033/044, 4. Überarbeitung.

[2] DIMDI 2020. Operationen- und Prozedurenschlüssel. Version 2020. Download unter: https://www.dimdi.de/static/de/klassifikationen/ops/ kode-suche/opshtml2020 (letzter Zugriff 08.06.2021)

[3] Abt-Zegelin A. Chronik einer Amputation - eine Patientin „fällt durch die Maschen“. Die Schwester - Der Pfleger 2011; 50: 34-37

$4 \quad$ Rehabilitation fängt im Grunde bereits vor bzw. bei der Amputation an. Leider sehen systembedingt die durchführenden Operateur*innen nur sehr selten Ihre Patient* innen nach der Rehabilitation bzw. meistens nur bei einer Revisions-OP. Sie sehen also nicht regelhaft ihr Operations-Endergebnis. Auch würden einige Operationen sicher anders durchgeführt, würde es vorher einen Austausch zwischen Orthopädietechnik, Rehabilitationsmedizin, Physiotherapie und (Gefäß-)Chirurgie geben. In anderen Ländern wie beispielsweise in Schweden wird eine solche interdisziplinäre Zusammenarbeit bereits praktiziert. 
[4] Destatis (2020). Gesundheit / Fallpauschalenbezogene Krankenhausstatistik (DRG-Statistik) / Operationen und Prozeduren der vollstationären Patientinnen und Patienten in Krankenhäusern (4-Steller). Wiesbaden.

[5] Spoden M. Amputationen der unteren Extremität in Deutschland - Regionale Analyse mit Krankenhausabrechnungsdaten von 2011 bis 2015. Gesundheitswesen 2019; 81: 422-430

[6] Destatis (2010). Gesundheit / Fallpauschalenbezogene Krankenhausstatistik (DRG-Statistik) / Operationen und Prozeduren der vollstationären Patientinnen und Patienten in Krankenhäusern. Ausführliche Darstellung. Wiesbaden.

[7] Spoden M, Nimptsch U, Mansky T et al. Amputation rates of the lower limb by amputation level - observational study using German national hospital discharge data from 2005 to 2015. BMC Health Serv Res 2019; 19: 8

[8] Michaelis U, Gaidys U.(2012). Abschlussbericht zur Studie “Optimierung der rehabilitativen Versorgung von Menschen vor, während und nach Majoramputation. Download unter: https://reha-vffr.de/images/ vffrpdf/projekte/2012/Bericht_final_2012_12_21.pdf (letzter Zugriff 10.05.2021).

[9] Gaidys U, Michaelis U. Optimierung der rehabilitativen Versorgung von Menschen mit Majoramputation - eine qualitative Studie. Pflege 2013; 26: 31-41

[10] DVfR (2013). Empfehlungen zur Verbesserung des teilhabeorientierten Versorgungsprozesses für Menschen mit Beinamputation unter besonderer Berücksichtigung der Prothesenversorgung. Download unter: https://www.dvfr.de/fileadmin/user_upload/DVfR/Downloads/ Stellungnahmen/DVfR-Empfehlungen_Versorgungsprozess_Amputation_-_Nov_Copy.pdf (letzter Zugriff: 28.07.2020).

[11] Egen C, Ranker A, Höpner K, Sturm C, Gutenbrunner C, Fink M, Banaschak H, Lemke S, Bethge M, Schiller J. Versorgungskonzept zur Schließung der rehabilitativen Lücke nach Majoramputation der unteren Extremität. Praxisbericht aus einem Innovationsfondsprojekt des Gemeinsamen Bundesausschusses. RP Reha 2020; 3: 27-37

[12] Gailey RS, Roach KE, Appelgate EB. The Amputee Mobility Predictor: An Instrument to Assess Determinants of the Lower Limb Amputee's Ability to Ambulate. Arch Phys MedRehabil 2002; 83: 613-627
[13] Granger CV, Brownscheidle CM. Outcome Measurement in Medical Rehabilitation. International Journal of Technology Assessement in Health Care 1995; 11: 262-268

[14] Bullinger M, Kirchberger I. SF-36 Fragebogen zum Gesundheitszustand. Göttingen: Hogrefe-Verlag; 1998

[15] Legro MW, Reiber GD, Smith DG, del Aguila M, Larsen J, Boone D. Prosthesis evaluation questionnaire for persons with lower limb amputations: assessing prosthesis-related quality of life. Arch Phys Med Rehabil 1998; 79: 931-938

[16] Deck R, Mittag O, Hüppe A, Muche-Borowski C, Raspe H. Index zur Messung von Einschränkungen der Teilhabe (IMET) - Erste Ergebnisse eines ICF-orientierten Assessmentinstruments. Praxis Klinische Verhaltensmedizin und Rehabilitation 2007; 76: 113-120

[17] Mittag O, Raspe H. Eine kurze Skala zur Messung der subjektiven Prognose der Erwerbstätigkeit: Ergebnisse einer Untersuchung an 4.279 Mitgliedern der Gesetzlichen Arbeiterrentenversicherung zu Reliabilität (Guttman-Skalierung) und Validität der Skala. Rehabilitation 2003; 42: 169-174

[18] Tuomi K, Ilmarinen J, Jahkola A et al. Arbeitsbewältigungsindex. Work Ability Index. Schriftenreihe der Bundesanstalt für Arbeitsschutz und Arbeitsmedizin, Band 14. Bremerhaven: Wirtschaftsverlag NW, Verlag für Neue Wissenschaft,; 2001

[19] Schmidt ], Lamprecht F, Wittmann WW. Zufriedenheit mit der stationären Versorgung. Entwicklung eines Fragebogens und erste Validitätsuntersuchungen. Psychother med Psychol 1989; 39: 248-255

[20] Simmel S, Müller WD, Reimertz C, Kühne C, Glaesener J]. Phasenmodell der Traumarehabilitation - Wie können wir das „Rehaloch“ vermeiden? Unfallchirurg 2017; 120: 804-812

[21] Hoffmann S. Entlassen in den Stillstand. Unfallchirurgen schlagen Alarm: Dank der Hochleistungsmedizin überleben immer mehr Schwerverletzte. Ein Großteil fällt nach der Akutbehandlung aber in ein Reha-Loch. Wie lässt sich das lösen? Frankfurter Allgemeine Sonntagszeitung 2020; 27: 17

[22] G-BA Innovationsausschuss (2016): Förderbekanntmachung vom 11. Mai 2016. 